\title{
LA TEORÍA DE IAA GRAMATICALIZACIÓN Y SUS APLICACIONES EN LAS LENGUAS ROMÁNICAS
}

\author{
BÉATRICE LAMIROY \\ K.U. Leuven \\ beatrice.lamiroy@arts.kuleuven.ac.be
}

\begin{abstract}
Resumen
The paper aims at showing how grammaticalization theory applies to Romance languages. In the first part of the paper we give a short overview of the main definitions and principles of the theory. Since grammaticalization phenomena regard all components of langualkge, we distinguish between semantic, syntactic, morphological and phonetic aspects of the process. Furthermorc, we also explore the question why this theory has been so successfull of late. Its success raises antoher question in turn, viz. whether we are dealing with a new 'paradigm' in linguistics, particular in the field of linguistic typology.

The second part is devoted to the study of empirical data taken from two different areas, auxiliary verbs on the one hand and prepositional clauses on the other, in three Romance languages, French, Italian and Spanish. We argue, first, with respect to auxiliaries, that an analysis in terms of grammaticalization is to be preferred over other competing analyses such as raising or transparency because the theory allows to account for heterogeneous data. Auxiliary verbs indeed do not constitute a homogeneous class. Second, from a comparative vantage point, it is shown that French displays many more grammaticalization symptoms than Spanish does. Italian seems to occupy an intermediate position, as far as auxiliaries are concerned. With respect to prepositional complements, we only take French and Spanish into account. Once more, we argue that French is much more advanced on the grammalicalization chain than Spanish is and provide evidence for our claim.
\end{abstract}

\section{INTRODUCCIÓN}

La finalidad de este artículo es ilustrar cómo la teoría de la gramaticalización se puede aplicar en las lenguas románicas. La ilustración se hará con dos ejemplos, relativos a las perífrasis verbales por un lado (ejemplo 1) y a los complementos esenciales de tipo preposicional por el otro. Estos últimos adoptarán la forma de un infinitivo o de una subordinada que $F^{\prime}$ (ejemplo 2):

(1) a. Se pone a llover

b. Il se met à pleuvoir

a. Max amenaza con dimitir

b. Max menace de démissionner 
Compararemos en el primer caso el castellano, el italiano y el francés, y en el segundo, el castellano y el francés sólo. Demostraremos en los dos casos que el francés es una lengua mucho más gramaticalizada que el español. La posición del italiano es, desde el punto de vista tipológico, intermedia entre el francés y el castellano.

De paso, en el caso de las perífrasis, argumentaremos a favor de la teoría de la gramaticalización, ya que existen otros modelos de análisis que a nuestro modo de ver no explican los datos de manera satisfactoria. Asi, el poder explicativo de la teoria no sólo permite dar cuenta de los datos empíricos por analizar sino que también los datos que se van a describir confirman la validez metodológica del modelo escogido. Antes de pasar al análisis de los datos, esbozamos primero brevemente las pautas más importantes de la teoría que hemos adoptado.

\section{LA TEORIA DE LA GRAMATICALIZACIÓN}

\subsection{Definición del concepto}

Como veremos a continuación, la noción misma de gramaticalización no se đefine siempre de la misma manera.

2.1.1. El primero en utilizar el término de gramaticalización fue Meillet (1912:131) quién le dió la siguiente definición: l'attribution chu caractère grammatical à un mot jadis autonome, es decir, la atribución del carácter gramatical a una palabra anteriormente autónoma. En el ejemplo (3a), en efecto, el sustantivo latín casa termina por adoptar una función gramatical de preposición en francés chez. Se observa pues una transición del componente léxico al componente gramatical ya que el sentido léxico de casa, si aun está parcialmente presente en (3b), desaparece completamente en el caso de (3c):

(3) a. lat. $N$ casa $>$ fr: Prep. chez

b. On va chez Jean = On va d̀ la maison de Jean= Vamos a casa de Juan

c. On observe ce comportement chez les grands singes $\neq$ à la maison des grands singes

'Se observa este comportamiento en los monos grandes' $\neq$ en casa de los monos grandes

Ejemplos similares son:

(4) a. fr. medio $S N$ beau coup $>$ fr. moderno Adv. beaucoup

'bonito golpe' 'mucho'

b. fr. medio SV main tenant > fr. maderno Adv. maintenant

'teniendo la mano' 'ahora'

c. esp. ant. SN vuestra merced > esp. moderno Pro Usted

(5) a. ingl. $N$ while > Conj. while

'momento' 'mientras que'

b. Let's stop for a while

'Paremos un momento' 
c. She was studying in an American college white her father in Iran was ill (Talmy 1988)

'Estaba estudiando en un colegio americano mientras que su padre estaba enfermo en Irán'

2.1.2. El estructuralista Kurylowicz (1965:52) dió una definición más amplia a la noción. Por gramaticalización se entiende: the increase of the range of a morpheme advancing from a lexical to a grammatical or from a less grammatical to a more grammatical status. En los ejemplos de (6) se trata cfcctivamente de elementos gramaticales del latín, un pronombre demostrativo y pronombre numeral, que se convierten en las lenguas románicas modernas en el artículo definido o un clítico de acusativo y un artículo indefinido respectivamente. Los últimos son más integrativos, tienen menos autonomía que los primeros, tal como se desprende de los ejemplos en (7): mientras illa podía funcionar como respuesta a una pregunta, tal no es el caso cuando se trata de la o un porque no tienen suficiente autonomía:
(6) a. lat. pron. illa > friespitit. clit. la
b. lat. num. unus > friespiti. artic. un
(7) a.lat. quis venit? illa
'quién vino? ella'
b. a quién viste? * la
c. qué comiste? un caramelo $/ * u m$

Definido de esta manera, el concepto evoca la transición del componente léxico al componente gramatical tanto como las transiciones que pucde haber dentro del componente gramatical mismo. Esto último tiene una consecuencia importante desde el punto de vista teórico: ya que un elemento puede estar más o menos gramaticalizado dentro del mismo componente, implica que la gramaticalización es fundamentalmente una cuestión de gradación (Heine 1993:49).

2.1.3. En tercer lugar, desde los trabajos de Traugott (1989, 1995; Traugott \& König 1991), la noción de gramaticalización se acerca a lo que se podría llamar una pragmaticalización. Por el hecho de adoptar un sentido más abstracto, la lengua adquiere nuevos medios lingüísticos, que se refieren menos al mundo concreto que nos rodea (por ejemplo las expresiones que indican relaciones espaciales) que a la organización del mundo por los hablantes, por medio de indicaciones de tiempo o de conexiones lógicas por ejemplo. Fue en este contexto en el que Traugott introdujo una noción nueva, la de subjectification: I have argued here that what is strengthened [pragmatic strengthening] is specifically the subjective stance of the speaker (Traugott 1995:48). Un caso concreto se da en las conjuncióncs temporales while o since en inglés que pasaron a ser utilizadas como conectores de oposición y de causa respectivamente. La transición de la categoría temporal a la categoría lógica se posibilita gracias al hecho de que ciertas inferencias hechas por el hablante o/y el interlocutor acaban por hacer parte del sentido mismo de las conjunciones:

(8) a. While her husband was doing the dishes, she was reading the newspaper $a^{\prime}$. Mientras (= en el momento en que) su marido lavaba los platos, ella leia el periódico [simultaneidad temporal] 
a". Mientras su marido lovaba los platos, ella leia el periodico

[inferencia posible: si el hablante presenta estas dos situaciones de forma simultanea, es que la simultaneidad es sorprendente, de lo cual se deduce un sentido adversativo]

(9) a. Since I left home, my mother has been mad at me (Traugott 1995)

'Desde que me fuí de casa, mi madre está enfaduda conmigo' [sucesividad temporall

b. Since I am leoving home, my mother is mad at me

'Como me voy de casa, mi madre está enfadada conmigo [inferencia posible: entre dos cosas que se suceden existe a menudo una relación de causa - cf. Ha llovido, el suelo está mojado]

Las expresiones, una vez gramaticalizadas, tambien pueden referirse al universo de creyencias subjetivas del hablante, por ejemplo a través del uso de los modales cpistémicos:

(10) a. Pablo debe dinero a sus padres [sentido concreto]

b. Pablo deberia dimitir [sentido deóntico]

c. Pablo debe estar enfermo [sentido epistémico $=$ Deduzco de $X$ que Pablo está enfermo]

\subsection{Síntomas de gramaticalización}

Todo proceso de gramaticalización implica que ciertas propiedades lingüísticas de las palabras se alteren. Las modificaciones abarcan todos los componentes del lenguaje, ycndo de la semántica a la fonética. Por lo tanto se pueden distinguir distintos parámetros que permiten detectar que un proceso de gramaticalización está teniendo lugar.

\subsubsection{Parámetro semántico}

Damourette \& Pichon (1911-1936) introdujeron el término de désémantisation para indicar lo que resulta ser una de las características más típicas de las palabras que se gramaticalizan, es decir, la pérdida de rasgos semánticos originales. En ese mismo contcxto se ha hablado de semantic bleaching 'empalidecimiento' (Lehmann (1985a, 1995, 2002), de light verbs o 'verbos ligeros', preposiciones incoloras, etc. En efecto, en (11a), el verbo ir cs un verbo de movimiento, mientras que en (11b) es un auxiliar o verbo ligero, en (11c) la preposición de aún indica el origen de un movimiento, mientras que en (11d) no es más que un mero complementizer:

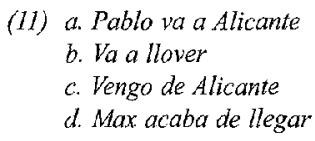

La desemantización de una palabra tiene a menudo como consecuencia una obligatorification (Lehmann 1985a), es decir, un condicionamiento mayor: mientras que escogemos 'libremente' entrc las palabras del léxico, existe cierta servidumbre respecto a las palabras gramaticales: 
(12) a. Va al cine con/sin vosotros

b. Cuento con/*sin vosotros

Cabe señalar que la descoloración del elemento gramaticalizado no equivale a un empobrecimiento en términos absolutos: las palabras se liberan para desempeñar otras funciones. Como lo ilustran (11a-b), pasan de la categoría del espacio a la del tiempo (Heine 1993, Lamiroy 1991, 1999), o como lo vimos en (8-9), se pasa de la cxprcsión del tiempo a la causa y a la concesión (Traugott 1988). Las palabras adoptan pues nuevas funciones, más abstractas, y contribuyen de este modo a la enorme economía del lenguaje: pocas formas por muchas funciones.

También hay que notar que toda desemantización no es sinónimo de gramaticalización. En el caso del uso metafórico de las palabras (de hecho otra manera de utilizar el lenguaje de forma económica ya que la misma forma cubre varios sentidos), el sentido original desaparece en parte pero no por eso se habla de gramaticalización:

(13) a. Veo a Juan
b. No veo lo que quieres decir

\subsubsection{Parámetro sintáctico}

Desde el punto de vista sintáctico, la gramaticalización conlleva lo que los teóricos del modelo han llamado una descategorialización (Hopper \& Traugott 1993): al perder parte de su significado, las palabras pierden también la capacidad de 'referir según su propia categoría'. Si los N y los V reficren típicamente, en términos generales, a entidades y situaciones, respectivamente, estas categorías, una vez gramaticalizadas, ya no disponen de los medios formales que permiten identificar sus referentes, in casu los morfemas de flexión para los V o los morfemas de número para los $\mathrm{N}$. Dicho de otro modo, pierden en parte los morfemas que normalmente actualizan los referentes. Mientras el verbo dc movimiento ir se emplea en todos los tiempos del indicativo, el verbo ligero, auxiliar del futuro, ir sólo se encuentra en el presente o el imperfecto de indicativo (14a), asímismo se observa que un sustantivo como gracia deja de declinarse cuando se utiliza como conectivo (14b):

$$
\begin{array}{ll}
\text { (14) a. Pablo valirálha ido/fue a Alicante } & \text { is Va/* irál* ha ido/ * fue a llover } \\
\text { b. la graciallas gracias } & \text { vs gracias/ " gracia a que Juan vino a la } \\
& \text { reunión se resolvió el problema }
\end{array}
$$

Otra consecuencia de la descategorialización es que la capacidad de subcategorización se debilita. En este sentido, las categorías léxicas mayores ( $\mathrm{N} \mathrm{y} \mathrm{V)} \mathrm{se} \mathrm{acercan,} \mathrm{al} \mathrm{gramati-}$ calizarsc, a las categorías léxicas segundarias (Adj, $\Lambda \mathrm{dv}$, Prep) que de por sí tienen menos capacidad de selección. Una propiedad bien conocida de los verbos auxiliares (perífrasis verbales) consiste en la ausencia de restricciones de selección en cuanto al sujeto, lo cual fue el argumento crucial de la gramática generativa para proponer un análisis por elevación de sujeto. En efecto, contrasta el sujeto necesariamente humano en (15a), cn donde se trata de un verbo de movimiento utilizado en su sentido literal, con los sujetos variados que pueden aparecer cuando el verbo ya no expresa el espacio (15b). Asimismo, el ejemplo (15c) 
sugiere que el sujeto de la expresión idiomática las narices viene seleccionado no por el verbo auxiliar sino por el infinitivo hinchar:

(15) a. Pablo ha venido a darme un beso esta manaña

b. Pablo/ el texto/ eso viene a decir lo siguiente

c. A Juan se le van a hinchar las narices

En cl caso de V, se observa un fenómeno que se puede interpretar de la misma manera: se trata de la pérdida de la capacidad predicativa. El verbo francés avoir permite una doble predicación cuando se utiliza en su sentido léxico lleno (16a), como auxiliar de los tiempos compuestos sin embargo, sólo una predicación es posible:

(I6) a. I'ai ma fille malade [tengo una hija + mi hija está enferma]

b. J'ai appelé ma fille [ he llamado a mi hija]

Asimismo se puede constatar que se pierden otras propiedades constitutivas de la categoría. En el caso de V, por ejemplo, la alternancia entre los auxiliares de los tiempos compuestos en lenguas como el francés o cl italiano viene determinado por el verbo. Sin embargo, en (17a-b), la alternancia essere vs avere en italiano viene determinado no por el verbo conjugado cominciare sino por el infinitivo siguiente. $Y$ como se ve en (18a-b), los sintagmas introducidos por las preposiciones de y a corresponden a formas pronominales de tipo acusativo, es decir, las preposiciones han perdido en este caso un rasgo definitorio de su categoría, o sea, la propiedad de construir complementos oblicuos:

(17) a. Ha cominciato a lavorare 'Ha empezado a trabajar'

b. Ė cominciata a cadere la poggia [E caduta la pioggia]

'Ha empezado a caer la llwia'

(18) a. Paul décide de partir/ Paul le décide

'Pablo decide marcharsel Pablo lo decide'

b. Pablo aprende a nadar / Pablo lo aprende

\subsubsection{Parámetro morfológico}

Fl proceso general de debilitación que padecen los clcmentos que se gramaticalizan tiene, como es lógico, un aspecto morfológico: por coalescence (Lehmann 1985a) se entiende el proceso según el que un elemento lingüístico tenderá tanto más a apoyarse en su entorno cuánto menos sustancia tiene. Estudios de indole tipologica han demostrado en efecto que existe una tendencia universal (Heine 1993, 1997) de los morfemas libres a evolucionar hacia elementos clíticos o afijos. Las lenguas románicas proporcionan ejemplos de este fenómeno en la fusión del sustantivo latin mente con el adjetivo que lo modifica, fusión que dió lugar a la creación de los adverbios con sufijo -mente, o en la fusión del auxiliar habere con el infinitivo, que resultó en la forma sintética del futuro simple: 
(19) a. lat. gentile mente [SN]
'de manera gentil'
b. lat. cantare habeo
'he de cantar'

Otros efectos a nivel morfológico de la gramalicalización son la condensation (Lehmann 1985a) ilustrada en (20) y el orden fijo de los morfemas: los morfemas libres tienen un orden fijo o no; los afijos tienen un orden fijo por definición (21):
(20) a. lat. per + ad $>\quad$ esp./ptg. para
b. lat. $a d, a b$, apud > fr.
(21) a. mente gentile vs gentile mente
b. gentilmente vs *mentegentil

\subsubsection{Parámetro fonético}

Por fin, un elemento que se gramaticaliza padece fenómenos de erosión fonética o attrition (Lehmann 1985a). Tanto el artículo como el pronombre clítico presentan tal proceso en francés y en italiano pero no en castellano (22a-b). Nótese que la erosión del verbo inglés go se da en el caso del auxiliar del futuro, pcro no cuando el verbo se utiliza con su sentido léxico de verbo de movimiento (22c):
(22) a. it.fr. l'uccello/l'oiseau
b. fr. je l'aime
c. I am going/* gonna to Alicante
vs esp. el ave
vs esp. lo amo
vs It's going to/ gonna rain

\subsection{Condiciones' de gramaticalización}

\subsubsection{La frecuencia}

Como fue observado por Bybee et al. (1994) y Bybee \& Hopper (2001), la frecuencia de un elemento es proporcional con la falta de sentido especifico y con la ligereza de su volumen formal. Existe pues una doble correlación: correlación entre valor informativo y frecuencia, en la medida en que les elementos gramaticales se utilizan más frecuentemente que los elementos léxicos, y correlación entre volumen fonético y frecuencia ya que las palabras más frecuentes son las más cortas.

Desde el punto de vista cognitivo, se ha de subrayar la importancia de la routinisation (Haspelmath 1999): los elementos que se gramaticalizan se 'banalizan' a la vez que su frecuencia aumenta, lo cual facilita su memorización.

1 Se trata de condiciones necesarias pero no suficientes. 


\subsubsection{Condición sémantica}

No todos los elementos léxicos se prestan al proceso de gramaticalización: de hecho es menos probable que un nombre como wallpaper 'papel de pared' (Traugott \&. Heine 1991) se gramaticalice que un sustantivo como lat. casa porque este último designa un concepto más básico que el primcro.

La investigación tipológica (Heine 1993, Heine et al. 1991, Hcine \& Rch 1984, Kuteva 2001, Lamiroy 1999, Lehmann 1986) enseña que los elementos que se gramaticalizan salcn de un número limitado de campos semánticus, tal como

- cl cspacio: Vmovimiento se convierten típicamente en Auxiliares de tiempo y aspecto, p.ej. ir, fr. aller, ingl. go, neerl. gaan

- la posesión: $N$ que indican una parte del cuerpo (posesión inalienable) se convicrten tipicamente en preposiciones, p.cj. de cara a, a espaldas de, fr. face à

- la intencionalidad: V de voluntad se convierten típicamente en auxiliares temporales y modales, p.ej. ingl. will, alem. sollen

- ciertas relaciones actanciales: la construcción refleja se convierte típicamente en estructura medio-pasiva, p.ej. los libros se venden en los supermencados hoy en dia

- etc.

Una hipótesis aqui sería que existe una relación entre buenos candidatos para un proceso de gramaticalización y ciertas restricciones de tipo semántico-cognitivo, debidas tal vez a una cuestión de prominencia perceptual (Talmy 1988).

\subsubsection{Condición 'estructural'}

Según Hopper \& Traugott (1993:124) una condición imprescindible para que sc de un fenómeno de gramaticalización es lo que llaman layering: es decir, el concepto de gramaticalización está vinculado al principio de coexistencia de varias capas dentro de la lengua, o sea distintas formas con significados similares que compiten entre ellas. En este sentido Hcine (1993) utiliza la noción de grammaticalization chains: la gramaticalización es un proceso contínuo, en el que hay nccesariamente fases de overlap, es decir, épocas en que varias formas concurrentes coexisten.

Desce un punto de vista diacrónico, existen dos posibilidades (Hopper 1991): $1^{\circ}$ specialisation, que se da cuando una de las expresiones acaba generalizándose y se convierte en la forma obligatoria (23a-b) o $2^{\circ}$ persistence, que ocurre cuando las dos formas (una más gramaticalizada, otra menos gramaticalizada) se mantienen $(23 \mathrm{c}-\mathrm{d})$ :
(23) a. lat clás. patri dare
'dar al padre'
b. fr: ant. ne pas' point' goute/ mie
vs lat. vulg. ad patrem dare
c En tu lugar, habria / hubiera ido
vs fr. ne pas/ point
d. Ana va a casa / Va a llover [ir $=$ Vmt vs Aux] 


\subsubsection{Condición tipológica}

Los síntomas de gramaticalización están evidentemente condicionados también por el tipo de lengua en la que se producen. Asi por ejemplo, los fenómenos de coalescence no se darán en una lengua analítica que no tiene afijos.

\subsection{Exito del modelo}

\subsubsection{Nuevo paradigma}

El éxito actual de la gramaticalización como modelo teórico parece indudable, de manera que cabe preguntarse si no nos encontramos en presencia de un nuevo paradigma metodológico. Tres aspectos parccen importantes en estc contcxto.

Primero, el aspecto empírico: el modelo permite analizar, clasificar y explicar una cantidad impresionante de datos. En efecto, las investigaciones de los tipólogos aquí citados han demostrado masivamente que un gran número de fenómenos en las lenguas más diversas se dejan analizar en términos de gramaticalización.

En segundo lugar, el aspecto teórico es importante por varias razones. Desde el punto de vista del cambio lingüístico, el modelo adopta una posición muy clara en cuanto a la dirección de las evoluciones históricas: las formas se gramaticalizan de manera irreversible (Haspelmath 1999, Lehmann 2002). Pero el modelo no sólo presenta una hipótesis ambiciosa sobre la diacronia de las lenguas en general sino que pretende también tener un poder explicativo desde el punto de vista sincrónico (Lehmann 1985b). Además, varios autores sc han empeñado en buscar una explicación cognitiva del cambio: entre las causas posibles que constituirían la base de todo proceso de gramaticalización se han mencionado la simplificación (Roberts 1993), la ley del menor esfuerzo (Newmeyer 1998), el principio de iconicidad (Givon 1971), e incluso la ley de la mano invisible (Keller 1994, Klausenburger 1999).

Por fin, cada paradigma metodológico tambićn ticne un aspecto institucional sin el que el modelo no se podría propagar. En el caso de la teoría de la gramaticalización se averigua también este aspecto: no sólo existen centros de investigación que han adoptado claramente el modelo, como la Universidad de Köln, o el Max-Planck Insititut en Leipzig, sino que igualmente abundan las publicaciones, las tesis doctorales y los congresos dedicados al tema.

\subsubsection{Razones del éxito}

Aunque forzosamente es especulativo intentar explicar el porqué del éxito, se pueden evocar una serie de factores que sin duda han contribuido a ello. Los enumeramos a continuación.

Como hemos mencionado anteriormente, la valor universal del modelo le da un enorme poder explicativo: cuántos más datos se dejan explicar dentro de un modelo determinado, más poderoso el modelo. Muchísimas lenguas efectivamente presentan fenómenos que se pueden explicar en el marco de esta teoría.

Otro factor importante es el carácter muy flexible del modelo que permite describir una serie de datos heterogéneos de una manera elegante, algo que un modelo muy formalizado como el minimalismo permite mucho menos. Nociones básicas de la teoría como los 
conceptos de overlap, o layering predicen la coexistencia de formas concurrentes e incluso contradictorias, es decir, al contrario de modelos como la gramática generativa o incluso el estructuralismo, no preconiza el modelo una visión homogénca de lenguaje. He aquí un punto de comparación entre la teoría de la gramaticalización y la teoria del prototipo (De Mulder 2001): los dos modclos, uno cn cl nivel de la gramática y otro en el nivel del léxico, reivindican poder analizar los hechos lingüísticos de manera dinámica.

En tercer lugar, el modelo es un modelo global en el sentido de que hace predicciones sobre todas las categorias léxicas, que pertenezcan a las categorías abiertas o a las cerradas y adcmás, permite dar cuenta precisamente de los pasajes de una categoría a otra, como lo ilustran los ejemplos dados en (1). También es un modelo global en otro sentido, es decir, Ia gramaticalización abarca a todos los niveles linguísticos, como hemos visto en los apartados 1 y 2 :

- el nivel fonético

- el nivel morfológico

- el nivel sintáctico

- el nivel semántico

- el nivel pragmático

En cuanto a este último, el modelo lo integra no sólo en cuanto a la descripción de los efectos (p.ej. la nocíon de subjectification de Traugott $(1988,1995)$ ) sino también cn la búsqueda de las causas: de manera muy general la gramaticalización podría explicarse por necesidades de tipo comunicativo (Heine 2002, Chafe 2002), tanto por un principio de economía (Keller 1994, Klausenburger 1999, Marchello-Nizia 2000) como por un principio de creatividad, llamado extravagance por Haspclmath (1999).

Cabe señalar también que el modelo prevé distintos tipos de gramaticalización, desde un nivel muy concreto (p.ej. la aparición de formas nuevas), hasta un nivel muy abstracto que afecta todo el sistema de la lengua (Marchello-Nizia 2001), pasando por la reorganización de ciertos paradigmas (p.ej. la conversión del adjetivo numeral en artículo indefinido en francés medio, cf. Carlier \& Goyens 1998).

Y por último, el modelo se presta a aplicaciones interdisciplinarias, e.o. de tipo psicolingïístico (Kuteva \& Sinha 1994).

\section{Aplicación en las lenguas románicas}

\subsection{Las perífrasis verbales}

3.1.I. La noción de verbo auxiliar: verbo 'transparente' (elevación) o verbo gramaticalizado?

Según la definición universal (Steele et al. 1981), un auxiliar (abreviado: AUX) es una categoría sintáctica especial con los rasgos siguientes: $1^{\circ}$ se trata de un constituyente, libre o afjo $2^{\circ}$ con un comportamiento morfosintáctico que lo distingue de todas las demás categorías y $3^{\circ}$ que expresa el tiempo o el aspecto o la modalidad (abreviado: TAM verbs).

En el caso de las lenguas románicas, AUX es un vcrbo cuyo rasgo distintivo consiste en el hecho de que su complemento verbal corresponde a un participio, un gerundio o un infinitivo, mientras excluye la subordinada sustantiva (Lamiroy 1994, 1999). 
Son varias las teorías que han sido propuestas en la literatura consagrada a los verbos auxiliares para explicar sus propiedades peculiares. A continuación recordamos dos de ellas, la teoría de la transparencia y el modelo generativista que propuso el análisis por elevación de sujeto. Fn el fondo, las dos tratan a los auxiliares como una clase homogénea y basan su análisis en el mismo hecho empírico, es decir, el hecho de que el sujeto del verbo auxiliar no viene seleccionado por el verbo auxiliar sino por el verbo siguiente. Según el modelo transformacional de Harris (1976), adoptado por Gross (1975) y Gaatone (1995), AUX es un verbo transparente porque no forma pantalla entre el sujeto y el verbo siguiente. Una manera espectacular de demostrar que la selección del sujeto depende efectivamente del segundo verbo se da en las expresiones idiomáticas, como en (15) repetido aquí como (24), donde las narices viene seleccionado por hincharse y en las estructuras impersonales, como en (25), donde el sujeto $i l$ vs ça se impone claramente por el verbo meteorológico. Es ésta también la razón por la que la gramática generativa propuso hace tiempo el análisis por la elevación de sujeto:

(24) a. A Juan se le están hinchando las narices

b. A Juan se le van a hinchar las narices

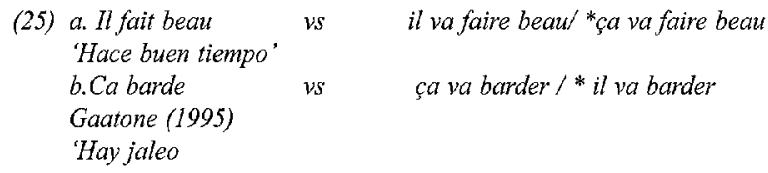

Basándose en el criterio del sujeto impersonal, Gaatone (1995) propone la siguiente lista de AUX para el francés:

(26)

$\begin{array}{llll}\text { aller } & \left(s^{\prime}\right) \text { arrêler } & \text { s'avérer } & \text { avoir béau } \\ \text { avoir failli } & \text { avoirl'air } & \text { cuvir manqué } & \text { cesser } \\ \text { commencer } & \text { continuer } & \text { devoir } & \text { être censé } \\ \text { ètre en passe } & \text { être en train } & \text { être en voie } & \text { ètre loin } \\ \text { être près } & \text { être supposé } & \text { être sur le point } & \text { finir } \\ \text { n'en pas finir } & \text { ne pas laisser } & \text { ne pas manquer } & \text { menacer } \\ \text { ne saurait } & \text { parấtre } & \text { pouvoir } & \text { risquer } \\ \text { se mettre } & \text { se révéler } & \text { sembler } & \text { tarder } \\ \text { tendre } & \text { se trouver } & \text { venir } & \end{array}$

Sin embargo, al averiguar si efectivamente todos los verbos de la lista admiten un sujeto impersonal, tal como lo predice la teoría de la transparencia, uno se da cuenta de que no es cstc cl caso. Como lo ilustra (27), solamente ir es compatible con el verbo impersonal falloir, mientras que los verbos estar a punto o se mettre producen frases inaceptables cuando se combinan con falloir:

(27) a. Il faut appeler un médecin

'Hay que llamar a un médico'

b. Il va falloir appeler un médecin

'Va a haber que llamar a un médico'

c. *Il est sur le point de'se met à falloir appeter un médecin

'Está a punto de ise pone a haber que llamar a un médico' 
(28) a. Hay un malentendido

b. Podria haber un malentendido

c. * Está a punto de/se pone a haber un malentendido

Por consiguiente, los análisis por transparencia o por elevación de sujeto fallan al predecir que la clase de AUX tiene un comportamiento homogéneo. En cambio, la teoría de la gramaticalización hace una serie de predicciones que se averiguan a nivel de los datos. En efecto, la clase de AUX es forzosamente heterogénca ya quc todos los verbos no se sitúan en el mismo sitio en la cadena de gramaticalización $o$, dicho de otro modo, algunos verbos están más gramaticalizados que otros. Dado que un proceso de gramaticalización implica la desaparición progresiva de las restricciones de selección, tal como lo hemos señalado en el apartado 2.2.2., es lógico que ciertos verbos, pero no todos, admitan cualquier tipo de sujeto: los más avanzados, como ir, efectivamente ya no imponen ningún tipo de restricción en cuanto al sujeto pero verbos monos gramaticalizados como fr. être sur le point, se mettre o esp. estar a punto, ponerse siguen imponiendo algún tipo de restricción puesto que no admiten un sujeto impcrsonal (27-28).

Asimismo se observa que los hechos ilustran otros principios previstos por la teoría de la gramaticalización, como la pérdida progresiva de la capacidad de subcategorización o la pérdida progresiva de propiedades típicas de la categoría. Esta permite en efecto explicar cómo ciertos verbos, pero no todos, admiten solamente un infinitivo mientras otros admiten un sintagma nominal también (29) y porqué ciertos verbos, pero no todos, admiten por ejemplo la forma del imperativo (30) o la negación del infinitivo (31):

(29) a. Empezamos a trabajarila lectura del libro

b. Acabamos de verlo/*de la vista

(30) a. Para de trabajar!

b. * Acuba de trabajar!

(31) a. Puede no ir a la fiesta

b. * Va a no ir a la fiesta

En resumen, la clase de AUX presenta une heterogeneidad que la teoría de la gramaticalización permite explicar ya que una de sus premisas mayores consiste en predecir fluctuaciones, episodios progresivos y características contradictorias. Como se ha podido obscrvar, cicrtos verbos están completamente gramaticalizados mientras otros lo están mucho menos.

\subsubsection{Comparación de las lenguas románicas}

\subsubsection{Tamaño de la clase de $A U X$}

Si uno compara la clase de verbos que entran en perífrasis verbales en francés, italiano y castellano ${ }^{2}$, se constata una diferencia significativa en cuanto al número de verbos. Son 73

2 Rccordcmos que el criterio formal es la ausencia de la subordinada sustantiva y el criterio semántico el de expresar el tiempo, el aspecto o el modo. 
verbos en francés (tabla 1 de Gross 1975), 115 en italiano y 171 en español (Lamiroy 1991). El italiano y el castellano tienen efectivamente auxiliares aspectuales cuyo significado se expresa por medio de cxpresiones adverbiales en francés:

$$
\begin{array}{ll}
\text { (32) a. Juan suele pasar por aqui } & \text { is Jean passe d'habitude par ici } \\
\text { b. I Torna a piovere } & \text { vs Il pleut de nouveau }
\end{array}
$$

También es cierto que los incoativos tienen más sinónimos en italiano y en castellano que en francés ${ }^{3}$ : mencionemos por ejemplo I acconciarsi (prepararse), apprestarsi (prepararse), avviarsi (estar a punto), buttarsi (echarse), (in)cominciare (empezar), mettersi (ponerse), scoppiare (explotar), y $\mathbf{E}$ arrojarse, comenzar, echarse, empezar, encaminarse, meterse, ponerse, romper, soltarse, saltar, etc:

(33) a. I Poi però scoppiarono a piangere (V. Brancati, tomado de Moretti \& Orvieto 1984: 105)

'Pero después se echaron a llorar'

b. Ana rompió a llorar

Asimismo se observa que el número de preposiciones que pueden introducir el infinitjvo es mayor en italiano y en castellano que en francés. Nótese que en los dos aspectos, en cuanto a número de verbos y a número de preposiciones, es el castellano el que domina. El francés utiliza las preposiciones $\dot{a}$, de, par, el italiano $a$, di, per, in, con y el castellano $a$, de, por, en, con, $\sin ^{4}$ :

(34) a. Amenaza con llover

b. I L'anima di lui che (...) aveva finito con tingersi di rousseauiani scrupoli (G.T. di Lampedusa tomado de Moretli-Orvieto 1984:100)

'El alma de quién había terminado por teñisse de escripulos à la Rousseau'

Y por fin, el tipo de aspectuales es más variado en italiano y en castellano que en francés. Asi por ejemplo hay iterativos que no tienen su pendiente en francés: I costumare (solcr), solere (soler), tornare (volver) E acostumbrar, avezar, soler, volver (cf. (32)). También los verbos que indican la velocidad con la que se efectúa una acción son raros en francés pcro menudean en las dos otras lenguas románicas: $\mathbf{F}$ se dépêcher vs tarder $\mathbf{I}$ affrettarsi (darse prisa), premurarsi (darse prisa), spicciarsi (darse prisa), sbrigarsi (darse prisa) vs trattenersi (entretenerse), indugiare (tardar) E apresurarse, apretar, apurarse vs entretenerse, atrasarse, demorar(se), retrasarse, tardar:

\section{(35) a. I Spicciamoci a mangiare \\ 'Comamos de prisa' \\ b. Max se entretwo mucho en explicar lodos los detalles}

Aquí se podrian mencionar también los verbos cuyo scmantismo indica la dificultad o la intensidad con la que se efectúa una acción: I agognare (morirse), faticare (cansarse), stiuggersi (ajetrearse) $\mathbf{E}$ afanarse, agobiarse, ajetrearse, chiflarse, morirse, perecerse:

3 Para más ejemplos véasc Lamiroy (1999).

4 Para más ejemplos véase Lamiroy (2001). 
(36) a. I Si struggeva di potersi sdebitare (Renzi \& Salvi 1991:531)

'Se ajetreaba por poder devolver sus deudas'

b. E Ana se agobia por educar a sus hijos

\subsubsection{Síntomas de descategorialización}

Tal como hemos mencionado en el apartado teórico (2.2.2.), la capacidad de subcategorización disminuye a medida que un verbo se gramaticaliza. El hecho de que algunos verbos aun admitan un SN como complemento, al lado del infinitivo, se puede interpretar como un signo de que el verbo no está del todo gramaticalizado. Pues, lo que se observa es quc esta alternancia V-inf / SN es más bien esporádica en francés (menos de un tercero de todos los verbos de la tabla 1 de Gross 1975), mientras afecta en italiano un poco menos de la mitad y en castellano más de la mitad de los verbos (Lamiroy 1999). O dicho de otro modo, el castellano resulta en este sentido, una vez más, la lengua menos gramaticalizada de las tres:

(37) a. Max se detuvo mucho en comeniar los hechos/el comentario de los hechos b. F Max tarda longtemps à expliquer les faits/* dans l'explication des faits c. I Lei si diede a raccogliere attomo le provviste avanzate (N. Ginzburg, tomado de Moretti \& Orvieto 1984: 104)/Lei si diede allo studio dei verbi servili 'Ella se puso a recoger alrededor las provisiones deterioradas / Ella se puso al estudio de los verbos auxiliares'

De manera análoga observamos que muchos de los verbos que en italiano y cn castellano pertenecen a la clase de los verbos auxiliares (porque no admiten la subordinada sustantiva y son desde el punto de vista semántico verbos de tipo TAM) aún seleccionan un sujeto de tipo Nhum, lo cual sc pucde considerar como un signo del bajo nivel de gramaticalización, ya que un verbo totalmente gramaticalizado típicamente admite cualquier especie de sujeto, humano o no, personal o no, nominal o no, etc.:

\section{(38) a. I* Si strugge di piovere \\ b. $E *$ Se ajetrea por llover}

O dicho de otra manera, estos verbos han empezado un proceso de gramaticalización, pero no lo han llevado a término.

Teniendo en cuenta las propiedades examinadas aquí, respecto no solo al número de verbos de la clase AUX sino también a las características seleccionales de los verbos, podemos concluir que de los tres idiomas estudiados, el francés es cl que resulta tener el grado más avanzado de gramaticalización. A continuación veremos que lo que acabamos de constatar en cuanto a las perifrasis verbales, también vale para los complementos preposicionales de tipo Prep V-inf o Prep Que F. 


\subsection{Los complementos preposicionales}

\subsubsection{El francés}

\subsubsection{El infinitivo preposicional}

Dentro de los complementos esenciales del verbo, se distinguen los sintagmas preposicionales cuyo núcleo es de tipo nominal y los de tipo frástico, es decir construidos por la preposición que rige un infinitivo o una subordinada en que. Lo que es caractéristico del francés es que el número de preposiciones susceptibles de introducir un complcmento (esencial) preposicional, varía según el tipo. Cuando se trata de un complemento cuyo núcleo es nominal, preposiciones como sur, dans, en, pour cuentan entre las posibilidades (39a-b-c). Pero cuando cl complemento es un infinitivo (40a-b-c), las preposiciones se reducen necesariamente a cero o a las preposiciones à o de (Grevisse-Goosse 1994: 1282-1298; Le Goffic 1993: 337; Lasserre 1976; Riegel et al. 1996:337, 495):

(39) a. Il compte sur vous

'Cuenta con vosotros'

b. Il se complait dans son malheur

'Se complace en su desdicha'

c. Le probleme consiste en ceci

'El problema consiste en esto'

(40) a. Il compte pouvoir partir ce soir

'Cuenta con poder marcharse esia noche'

b. Il se complât à répéter la même histoire

'Se complace en repetir la misma historia'

c. Le jeu consiste à marquer des points le plus vite possible

'El juego consiste en marcar puntos cuanto antes'

Este hecho se puede relacionar con una de las características del proceso de gramaticalización que hemos esbozado en el apartado teórico (2.2.1.), es decir, lo que Lehmann (1986, 1995) ha llamado obligatorification: la reducción de las posibilidades de escoger entre los clemcntos de un paradigma corresponde en efecto a una especialización.

Por otra parte, el hecho de que sólo dos preposiciones sirvan para introducir la totalidad de los complementos infinitivos sugiere que éstas se han desemantizado: son efectivamente incoloras o abstractas (Spang-Hanssen 1963, Kilroc 1991, Cadiot 1997).

Otro síntoma de gramaticalización que hemos señalado anteriormente se observa aqui: en efecto, a pesar de la presencia de una preposición, el complemento infinitivo de muchos verbos corresponde a un clítico directo, de tipo acusativo. Por lo tanto, la preposición deja de construir un complento indirecto o oblicuo, lo cual se puede interpretar como un rasgo de descategoralización.

(41) a.Max accepte/craint/essaye'proposetregrette/refise d'y aller 'Max aceptafteme/intentafpropone/siente/rechaza ir ahi' b. Max l(e) accepte/craint/essuye/propose'regrette'refuse 'Max lo acepta /teme/intenta/propone/siente/rechaza' 
Notemos por último que la preposición de presenta otra señal, fonética en este caso, de gramaticalización: encontramos la forma con erosión d' ante vocal, tal como lo ilustra el ejemplo (41).

\subsubsection{La subordinada Prep Que F}

Desde el punto de vista normativo, no sólo las preposiciones à y de, pero también en y sur pueden introducir una subordinada en francés: Ricgel et al. (1994: 493) y Le Goffic (1993: 343) mencionan à ce que, de ce que y en ce que, Goosse-Grevisse (1994:1601) añade sur ce que:

(42) a. La difficulté consiste en ce que nous avons peu de temps (Le Goffic (1993: 343) 'La dificultad consiste en que tenemos poco tiempo'

b. Il insiste beaucoup sur ce que ces deux imprudentes n'ont pas emmené de chauffeur (Mauriac, tomado de Goosse-Grevisse (1994:1601)

'Insiste mucho en que estas dos impridentes no se han llevado un choffer'

Hay que señalar sin embargo que Gross (1975: 181) clasifica insister como un verbo que rige una subordinada directa, es decir de tipo que $F$ y no Prep que F. Señalemos asimismo que Grevisse-Goosse (1994: 1601) indican sur ce que como posible conjunción pcro quc dan como ejemplo para compter sur una frase con subordinada directa, es decir, de tipo que $F$ y no Prep que F:

(43) Je comptais que vous viendriez à mon aide (Grevisse-Goosse 1994: 1601) 'Contaba con que Usted vendría a ayudarme'

Lo que se desprende de estos datos, a nuestro modo de ver, es que la subordinada preposicional indirecta (ya?) no es totalmente natural en francés, por lo menos no con las preposiciones en y sur.

Si fuera verdad que las conjunciones en ce que y sur ce que están actualmente retrocedicndo en francés (cf. infra), esto podría interpretarse, una vez más, como una señal de gramaticalización, ya que vamos en el sentido de una reducción del paradigma, o de una especialización.

Para averiguar la hipótesis que acabamos de esbozars, hemos efectuado una investigación de una muestra de los verbos insister; compter, résider, consister a partir del corpus Frantext. Todos estos verbos rigen en principio una subordinada preposicional introducida por en ce que o sur ce que. El material investigado cubre los textos en prosa (novelas y cnsayos) del siglo XX. Esta pesquisa lleva a la conclusión siguiente: $1^{\circ}$ las dos conjunciones son poco frecuentes $2^{\circ}$ en ce que es más frecuente que sur ce que (sur ce que siempre aparccc con insister, en ce que se utiliza 7 veces con résider, 41 veces con consister) y $3^{\circ}$ si se compara la primera mitad del siglo con la segunda, las dos parecen en retroceso desde principios de siglo:

5 Evidentemente, sería preciso estudiar en un corpus mucho más grande las frecuencias relativas de las dos estructuras, con infinitivo y con subordinada respectivamente, para poder averiguar sistemáticamente nuestra hipótesis. 
(45)

\begin{tabular}{|l|l|l|}
\hline & en ce que & sur ce que \\
\hline $1900-1950$ & 33 casos & 3 casos \\
\hline $1950-2000$ & 15 casos & 0 casos \\
\hline
\end{tabular}

He aquí unos ejemplos tomados del corpus de Frantext anteriores incluso al siglo XX (47a-b): estas frases parecen efectivamente poco naturales hoy en día. El ejemplo (47c) de principios del siglo XX pertenece a un registro puramente escrito:

(46) a. La distinction de supériorité et d'infériorité consiste en ce que la mari a puissance sur la fernme, el la fernme est subjecte au mari (P. Charron. 1601. De la sagesse)

$b$. Toute la différence consiste en ce que la vie des femmes est un développement contimuel de leurs moeurs (J.J. Rousseau. 1758. Lettres à M. D'Alembert)

c. Son fondateur avait bien insisté sur ce que la presse entendait, malgré son bas prix, être un journal aussi bien et mieux fail que les autres (J. Morienval. 1934. Créateurs de la grande presse)

En cuanto a las conjunciones à ce que y de ce que, Le Goffic (1993:343) nota que la subordinada preposicional tout court parece «souvent recherchée, peu naturelle par rapport au complément infinitif»:

(47) a. Il a réussi à ce que tout soit fini à temps (Le Goffic (1993:343)

b. Il a réussi à tout finir à temps

Efectivamente, muchas frases introducidas por à ce que, de ce que parecen bastante menos aceptables que las frases correspondientes con infinitivo:

(48) a. Paul rougit d' avoir dit cela

b.?? Paul rougit de ce qu'il ait dit cela

c. Mux a convaincu Paul de partir

d. ?? Max a convaincu Paul de ce qu'il parte

Que el infinitivo parezca más natural que la subordinada sustantiva podría formar parte del mismo proceso generalizado de gramaticalización al que asistimos en francés. El infinitivo está sintácticamente más integrado en el SV que la subordinada (el sujeto es un elemento vacío $\mathrm{PRO}$, el verbo es una forma no tensada), y a la vez es morfológicamente y fonéticamente más ligero, por lo tanto más coalescente que la subordinada. Según los cspecialistas del francés hablado (Claire Blanche-Benveniste, comunicación personal) Prep que $P$ haría parte de los fenómenos que se evitan en el lenguaje oral, lo cual parcce altamente significativo ya que los cambios lingüísticos, como bien se sabe, se originan en la lengua hablada antes de pasar a la lengua escrita. Cabe señalar también que Gross (1975), que considera muchas subordinadas preposicionales como aceptables en francés, utilizaba la noción de frase teórica, es decir, frases posibles dentro del sistema de la lengua pero raramente realizadas por los hablantes. Por supuesto los dos tipos coexisten actualmente, el infinitivo y la subordinada preposicional, de manera que se aplica lo que los teóricos llaman un estado 
de layering. Pero si lo que acabamos de esbozar aquí fuera cierto, se podría hablar de un principio de 'especialización'.

\subsubsection{El español}

\subsubsection{El infinitivo preposicional}

En castellano, las preposiciones que pueden introducir un complemento esencial no varian según que el complemento contiene un sustantivo o un infinitivo como núcleo. En ambos casos pueden ocurrir a, de, con, en, por (Cuervo 1994, Delbecque et Lamiroy 1999, Skydsgaard 1977):

(49) a. Max insiste en la urgencia del asunto

b. Ana se ajetrea por cosas initiles

c. Contamos contigo

(50) a. Victor balbuceú algo, como si no atinara a responder (Goytisolo, tomado de Skydsgaard 1977:518)

b. Max amenaza con abandonar a su familia

c. El cocinero se jactaba de guisar cualquier plato sin echarse la menor mancha (Unamuno, tomado de Cuervo 1994: verbo jactarse)

Esto significa que el castellano, contrariamente a lo que hemos observado para el francés (3.2.1.), no presenta en este caso cl sintoma de gramaticalización descrito arriba como especialización, dado que el número de preposiciones susceptibles de introducir el complemento es constante. En castellano tampoco se obscrva, en el caso de la preposición de, lo que hemos interpretado en el apartado anterior como una señal de descategoralización, es decir el hecho de que el complemento introducido por de se pronominalice en un clítico de tipo acusativo lo. Este fenómeno sólo se encuentra en el caso de la preposición a (51b). En los demás casos (51a), el clítico lo correspondo a un complemento directo:

(51) a. Max acepta/teme/intenta/propone/siente/rechaza / $y^{*} d e$ marcharse $=$ Lo aceptal...

b. Max aprende a nadar $=$ Lo aprende

Notemos por último que la preposición de no presenta el fenómeno de elisión que hemos registrado para la preposición equivalente del francés.

\subsubsection{La subordinada Prep Que F}

Al opuesto de lo que hemos constatado para el francés, las preposiciones que pueden introducir subordinadas en castellano son las mismas que las que introducen un complemento cuyo núcleo es nominal o de tipo infinitivo. Además, Prep que $F$ corresponde a la subcategorización de una gran serie de verbos: 192 verbos se construyen con a que $F, 102$ con de que $F, 46$ con en que $F, 31$ con por que $F$ y 17 con con que F (Delbecque \& Lamiroy 1999: 2076). 
(52) a. El problema radica en que en una gota de sangre hay mucha más información que la necesaria para una identificación segura (corpus El Pais, Goethals 2000)

b. Nada más llegar a la alcaldía me encontré con que la compañia eléctrica pretendia cohrar de un golpe (corpus El Pais, Goethals 2000)

c. Pero (...) Aznar ha optado por que todos los cambios sean compensados, con la única excepción del presidente del Senado, Juan Ignacio Barrera (corpus El Pais, Goethals 2000)

De los datos que acabamos de presentar se desprende que la subordinada preposicional cstá totalmente viva en castellano, mucho más que en francés donde solamente dos preposiciones, $\grave{a} \mathrm{y} d e$, introducen aclualmente subordinadas indirectas. Recordemos que incluso las subordinadas de tipo à ce que y de ce que parecen a menudo poco naturales, comparadas con los infinitivos correspondientes. Que el número de prcposiciones que introducen el complemento indirecto, que sea de tipo Prep $V$-info de tipo Prep que $F$ sea mayor en castellano que en francés sugiere que las preposiciones sc han especializado menos en español ya que especialización implica reducción. Teniendo en cuenta todo lo dicho anteriormente, se pucde concluir que el español cstá mcnos gramaticalizado que el francés.

\section{CONCLUSiÓN}

Llcgados al término de este artículo, pensamos haber demostrado que el interés de la teoria de la gramaticalización es doble, empírico y teórico a la vcz.

Por un Iado, el modelo permite contrastar desde el punto de vista tipológico entre lenguas más o menos gramaticalizadas, lo cual se aplica perfectamentc cn el caso de las lenguas románicas. Tanto el estudio de los auxiliares como el de los complementos preposicionales lleva a la conclusion, sin que sepamos exactamentc porqué, de que el francés es la lengua que está más avanzada en el proceso de gramaticalización y el castellano la que menos. El estudio de las perífrasis verbales ha revelado por otra parte que el italiano parece ocupar una posición intermedia. Evidentemente habría que ampliar el número de lenguas, incluyendo el portugués y el rumano para llegar a una vista más global del estado de gramaticalización de las distintas lenguas dentro de la familia románica. Pero la discreta investigación que acabamos de presentar permite por lo menos esbozar una hipótesis central según la cual el francés encabezaría la lista en cuanto al avance del proceso.

Por otro lado, la teoría que hemos adoptado aquí resulta teóricamente satisfactoria porque permite analizar datos que resisten a un análisis global o homogénco, tal como lo hemos demostrado en el caso de las perífrasis verbales. Como bien se sabe, los modelos que son compatibles con el mayor número de datos analizados son preferibles a los modelos más ambiciosos y a veces teóricamente más elegantes, pero que solamente se aplican a una serie limitada de datos.

\section{REHERENCIAS BIBLIOGRÁFICAS}

Bai Zeev Shymkioi, H. \& S. Kemm er (1995): «La gram maticaisaton des p répositions: concurrence f.T suinstitution》, Revue Romane, 30, 2, 206-224.

Bolinger, D. (1980): «Wanna and the gradience of auxiliaries». En Brcttschneider, G. \& C. Lehmann (dirs.): Wege zur Universalienforshung. Sprachwissenschaftiche Beiträge zum 60. Geburtstag von H. Seiler. Tübingen, G. Narr, 292-299. 
Bybee, J. \& P. Hopper (2001): Frequency and the Emergence of Linguistic Structure. Amsterdam, J. Benjamins.

Bybee, J., R. Perkins \& W. Pagliuca (1994): The Evolution of Grammar. Chicago, Chicago University Press.

Cadiot, P. (1997): Les prépositions abstraites en français. Paris, A. Colin.

Carlier, A. \& Goycns, M. (1998): «De l'ancien français au français moderne: régression du degré zếro de la détermianation et restructuration du systèmes des articles». En Klein, J., B. Lamiroy \& J.M. Pierret (dirs.): Linguistique et Informatique.Cahiers de l'Institut de Linguistique de Louvain-laNeuve, 24, 3-4, 77-112.

Chafe, W. (2002): «Putting grammaticalization in its place». En Wischer, I. \& G. Dicwald (dirs.): New Reflections on Grammaticalization. Amsterdam, Benjamins, 395-412.

Cuervo, R.J. (1994): Diccionario de Construcción y Régimen de la Lengua Castellana. Bogotá, Instituto Caro y Cuervo, 8 vol. (primera cdición vol. 1 1886, vol. 2 1893, vol. 3-8 1994).

Damourette, J. \& E. Pichon (1911-1936): Des mots à la pensée.Essai de Grammaire de la Langue française. Paris, d'Artrcy.

Delbecquc, N. \& B. Lamiroy (1999): «La subordinación sustantiva». En Bosque, V. \& I. Demonte (dirs.): Nueva Gramática de la Lengua Española. Madrid, Espasa-Calpe, 1965-2081.

De Mulder, W. (2001): «La Linguistique Diachronique: grammaticalisation et sémantique du prototype», Langages, 130, 8-32.

Detges, U. (2000), «Time and truth: the grammaticalization of resultatives and perfects within a thcory of subjectification», Studies in Language, 24, 2, 345-377.

Gaatone, D. (1995):» Syntaxe et sémantique: le cas des verbes 'transparents'», Le langage et le texte. Hommages à A. Lorian, 55-71.

Givon, T. (1971): «Historical syntax and synchronic morphology: an archaeologist's field tripm, Chicago Linguistic Society, 7, 349-415.

Goethals, P. (2000): Las conjunciones causales explicativas en castellano. Universidad de Leuven, tesis doctoral.

Grevisse M. \& Goosse, $\Lambda$ (1994): Le Bon Usage . Gembloux, Duculot.

Gross, M. (1975): Méthodes en Syntaxe. Paris, Hermam.

Harris, Z. (1976): Notes du cours de syntaxe. Paris, Le Seuil.

Haspelmath, M. (1999): «Why is grammaticalisation irreversible ?», Linguistics, 39, 1043-1068.

Heine, B. (1993): Auxiliaries: cognitive forces and grammaticalization. Oxford, Oxford University Press.

Heine, B. (1997): Possession: cognitive sources, forces and grammaticalization. Cambridge, Cambridge University Prcss (Cambridge Studies in Linguistics, vol. 83).

Heine, B. (2002): «On the role of context in grammaticalizationm. En Wischer, I. \& G. Diewald (dirs.): New Reflections on Grammaticalization. Amsterdam, Benjamins, 83-101.

Heine, B. \& Reh, M. (1984): Grammatical Categories in African Languages. Hamburg, Helmut Buske.

Heine, B., Claudi, U. \& Hünnemeyer, F. (1991): Grammaticalization. A Conceptual Framework. Chicago, Chicago University Press.

Hopper, P. (1991): «On some principles of Grammaticalisation». En Trangott, E. \& B. Heine (dirs.): Approaches to Grammaticalisation. Amsterdam, Bcnjamins, 17-35.

Hopper, P. (1998): «Emergent Grammar». En Tomascllo, P. (dir.): The New Psychology of Language. London, L. Eribaum Associates.

Hopper, P. \& Traugott, E. (1993): Grammaticalisation. Cambridge, Cambridge University Press.

Kilroc, P. (1991): «The grammaticalization of French à». En Pagliuca, W. (dir.): Perspectives on grammaticalization. Benjamins, Amsterdam, 49-61.

Keller, R. (1994): Language Change: the Invisible Hand in Language. London, Routledge.

Klausenburger, J. (1999): «Can grammaticalization be explained invisible-handedly ?» En Schmid, 
M., J. Austin \& D. Stein (dirs.): Historical linguistics. Amsterdam, Benjamins.

Kurylowicz, J. (1965): «The evolution of grammatical categorics». Esquisses linguistiques. Fink, Münich, 38-54. (edición 1975)

Kuteva, T. (2001): Auxiliation. An inquiry into the nature of grammaticalization. Oxford, Oxford University Press.

Lamiroy, B. (1991): Léxico y gramática del español. Estructuras verbales de espacio y de tiempo. Barcelona, Anthropos.

Lamiroy, B. (1994): «Les syntagmes nominaux et la question de l'auxiliaritć», Langages, 115, 6476.

Lamiroy, B. (1999): «Auxiliaircs, langues romanes et grammaticalisation», Langages, 135, 33-46.

Lamiroy, B. (2001): «La préposition on français et en espagnol: une question de grammaticalisation?», Langages, $143,90-105$.

Lang, J. \& Neumann-Holz, 1. (1999): Reanalyse und Grammatikalisierung in den romanischen Sprachen. Tübingen, Niemeyer.

Lasserre, E. (1976): Est-ce A ou DE? Lausanne, Payot.

Le Goffic, P. (1993): Grammaire de la phrase française. Paris, Hachette.

Lehmann, C. (1985a): "Cirammaticalization and Linguistic Typology", General Linguistics, 26, 3 23.

Lehmann, C. (1985b): «Grammaticalization: Synchronic Variation and Diachronic Change», Linga e Stile, XX, 3, 303-318.

Lchmann, C. (1995): Thoughts on Grammaticalization. München, Lincom.

Lchmann, C. (2002): «New reflections on grammaticalization and lexicalization». En Wischer, T. \& G. Diewald (dirs.): New Reflections on Grammaticalization. Amsterdam, Benjamins, 2-18.

Marchello-Nizia, C. (2000): «Les grammaticalisations ont-elles unc cause ?», L'information grammaticale, 67, 3-9.

Marchello-Nizia, C. (2001): «Grammaticalisation et Evolution des systèmes grammaticaux», Langages $130,33-41$.

Meillet, A. (1912): «L'évolution des formes grammaticales). Linguistique Historique et Linguistique générale. Paris, Champion (edición 1958).

Moreti, G. \& Orvieto, G. (1984): Grammatica Italiana. Perugia, Benueci.

Newmeyer, F. (1998): Language Form and Language Funciion. Cambridge, MIT Press.

Renzi, L. \& Salvi, V (dirs.) (1991): Grande Grammatica di Consultazione, Vol. II. Bologna, Il Mulino.

Riegel, M., M. Pellat, M. \& R. Rioul (1994): Grammaire méthodique du français. Paris, PUF.

Roberts, I. (1993): «A formal account of grammaticalization in the history of Romance futurcs», Folia Linguistica Historica, 13, 219-258.

Sinha, C. \& T. Kuteva. (1994): «Spatial and non-spatial uses of prepositions: conceptual integrity across semantic domains). En Schwarz, M. (dir): Kognitive Semantik/Cognitine Semantics. Ergebnisse, Probleme, Perspektiven. Tübingen, G. Narr.

Skydsgaard, S. (1977): La combinatoria sintáctica del infinitivo español. Madrid, Castalia.

Spang-Hanssen, E. (1963): Les prépositions incolores du français moderne. Copenhague, Gads Verlag.

Stcele, S. et al. (1981): An Encyclopedia of $\Lambda U X$ : a study in cross-linguistic equivalence. Cambridge, MIT Press.

Talmy, L. (1988): «The relation of grammar to cognition». En Rudzka-Ostyn, B. (dir.): Topics in Cognitive Linguistics. Amsterdam, Benjamins, 165-205.

Traugott, E. (1988): «Pragmatic Strengthening and Grammaticalisation», Proceedings of the $14 \mathrm{lh}$ Annual Meeting of the Berkeley Linguistic Society, 406-416.

Traugott, E. (1995): «Subjectification in grammaticalisation». En Stein, S. \& S. Wright (dirs): Subjectivity and subjectivisation. Cambridge, Cambrdige University Press. 
Traugott, E. \& B. Heine (dirs.) (1991): Approaches to grammaticalisation. Amsterdam, J. Benjamins.

Trangott, E. \& E. König (1991): «The semantics-pragmatics of grammaticalization revisited». En Traugott, E. \& B. Heine (dirs.): Approaches to grammaticalisation. Amsterdam, Benjamins, 189218. 